Teppersche Arbeit enthält die Beschreibung des $\sigma^{7}$, nach welcher dasselbe kleiner und heller als das $q$ sein, schlankere Antennen und noch flachere Augen als dieses besitzen soll. Über die Lebensweise wird leider nichts mitgeteilt, aufser dafs die Grillen unter Steinen mit Ameisen vorkämen. Das mir von Prof. Wh e e l e r gütigst zugesandte Exemplar war bei Camponotus nigriceps Sm. gefangen worden.

\title{
Zur Bienenfauna \\ Neuguineas und der benachbarten Gebiete. (Hym.) Nachtrag II.
}

Von Dr. H. Friese, Schwerin i. M.

Ceratina macrocephala n. sp. q.

Wie C. hieroglyphica, die ja sehr variabel in Gröfse wie Färbung ist, aber viel grölser, Kopf von Abdomenbreite und mächtig entwickelt.

․ Schwarz, kaum gelblich behaart, Kopf breiter als Thorax, und auch ebenso grofs, glatt, glänzend wie das Mesonotum, Clypeus verkehrt $\perp$-förmig, Rand zweimal gebuchtet, Labrum so lang wie breit, vorn gerundet, schwach konkav, mit gelber Scheibe und einzelnen grofsen Punkten, Clypeusscheibe und Nebengesicht gelb, Kopf hinten erweitert und hier mit breiter gelber Linie, Gesicht beulig verdickt. Pronotum, Scutellum und Metanotum gelb, Area fein skulpturiert, aber kaum matter; Rand von Segment 1 mit kleinem, gelbem Fleck, zweites ganz schwarz, drittes bis fünftes mit schmaler gelber Randbinde, die mitten erweitert ist, sechstes breit, zugespitzt, schwarz, runzlig und körnig punktiert, zweites bis fünftes einzeln punktiert. Bauch schwarz, lang greis behaart. Beine schwarzbraun, mit gelbem Kniefleck, Femurende unten mit gelber Linie, Scopa gelblich, Calcar gelbbraun, Tarsen an allen Beinen lang gelbbraun beborstet. Flügel gelblich getrübt, Adern braun, Tegulae schwarz. - L. $13 \mathrm{~mm}, \mathrm{Br}$. (Abd.) $4 \mathrm{~mm}$.

1 o von der Insel Nias, R a a p leg. 1898, in der coll. Magretti. - Indischer Ozean.

Anthophora flava n. sp. $q$.

Anthophora flava steht der A. bombiformis Sm. nahe, ist aber überall dicht gelbfilzig behaart, auch das Abdomen auf Segment 2-3.

7. Schwarz, dicht gelbfilzig behaart, Kopf und Thorax dicht 
runzlig-punktiert, glänzend, Clypeus und Labrum gelb, jederseits an der Basis mit verschwommenem, gelbem Fleck, Mandibel gelb, Spitze stumpf rotgelb, Antenne braun, unten rotgelb, zweites Geifselglied sehr lang $=3+4+5+6$, drittes fast so lang wie viertes. Abdomen gelbfilzig, dicht anliegend behaart, Segmentränder kaum bandiert erscheinend, fünftes nur mitten, sechstes ganz braun behaart. Ventralsegment punktiert, breit braunhäutig am Rande, lang gelblich gefranst. Beine gelblich, dicht gelb behaart, Scopa gelb, auch der Pedicellus gelb, nur Metatarsus innen braun behaart, Calcar gelb. Flügel hyalin, Adern braun, Tegulae gelb. - L. $15 \mathrm{~mm}, \mathrm{Br} .6 \mathrm{~mm}$.

$1 q$ von Freemantle. - West-Australien.

$$
\text { Anthophora rufescens n. sp. or. }
$$

Wie A. bombiformis Sm., aber Abdomen rot gefärbt, dünn rotgelb befilzt, ohne Binden.

๙7. Schwarz, gelbbraun behaart, Kopf und Thorax dicht runzlig punktiert, glänzend, Clypeus gelb, mit 2 grofsen eckigen braunen Basalflecken, Nebengesicht gelb, Labrum und Mandibel gelb, Mandibelende schwarzbraun. Antennenschaft vorn gelb, Antenne braun, zweites Geifselglied $=3+4$. Mesonotum - Behaarung mit zahlreichen schwarzen Haaren gemischt. Abdomen rot gefärbt, rotgelb anliegend befilzt, Segment 2 mit grofsem schwarzen Scheibenfleck, Segment 7 klein, kurz dreizähnig, die beiden Seitenzähne am längsten. Ventralsegmente gelb, punktiert, gelb gefranst, Segment 3-4 mitten eckig vorgezogen, 4 hier mit braunem Filzbüschel, 5 mit Mittelfurche, am Ende ausgerandet, 6 jederseits mit braunen Haarbüscheln. Beine rotgelb, gelblich behaart, Tibie III innen und Metatarsus unten schwarz behaart, Calcar gelb. Flügel hyalin, Adern braun, Tegulae gelblich. L. $13-14 \mathrm{~mm}$, Br. $5 \mathrm{~mm}$.

1 ๙ von Mackay, im März 1899, G. Turner leg. Queensland, Nordost-Australien.

Bestimmungstabelle für die Varietäten der Anthophora zonata F. (1858.)

\section{궁.}

1. Abdomen schwarz gefärbt. . . . . . . . . 2 .

Abdomen rot gefärbt. A. rufescens Friese.

2. Abdomen wie der Thorax meist gelb oder rotgelb dicht behaart . . . . . . . . . . . . . 3 .

Abdomen mit hellen Binden (blau, grün, weifs, goldig) 4. 
Abdomen wie der Thorax dunkelbraun behaart, Segment 4-6 goldglänzend behaart. A. aurata Friese.

Körper überall grünlichgelb befilzt. A. aeruginosa $\mathrm{Sm}$.

3. Körper rotgelb behaart, Segment 2-3 mit schwarzer Basis, Scopa rotgelb. A. bombiformis $\mathrm{Sm}$.

Körper gleichmäfsig gelb befilzt, Scopa gelb. A. flava Friese.

4. Thorax rotgelb behaart . . . . . . . . . 5 .

Thorax gelblich bis weifslich oder bläulich- bis aschgrau behaart.

A. cingulata $\mathrm{F}$.

Thorax schwarz behaart.

A. atrocaerulea Dours.

5. Segmentbinden blaugrün metallisch

Segmentbinden goldig glänzend, 4-6 gelbbefilzt.

A. flammeozonata Dours.

Segmentbinden weilslich, fast ohne Metallglanz.

A. pulchra Sm. (= fraterna Bingh.).

Segmentbinden rotbraun, Beine rotbraun, Scopa goldgelb.

6. Scopa weifslich.

Scopa rotgelb.

A. vigilans $\mathrm{Sm}$.

A. cincta $\mathrm{F}$.

A. zonata F.

(elegans Sm. 58, emendata Sm. 1879).

Scopa "nigro-ferrugineo pilosis". A. atrocaerulea Dours.

Anthophora a urata n. sp. or.

Durch die goldglänzende Behaarung von Segment 4-7 auffallend.

$\sigma^{7}$. Schwarz, schwarzbraun behaart, stellenweise auch gelbbraun behaart; Kopf und Thorax fein runzlig-punktiert, fast matt, Gesicht gelb, und zwar: Clypeus bis auf den länglichen braunen Fleck, jederseits der Basis, Nebengesicht, Antennenschaft vorn, Labrum bis auf die beiden runden braunen Basalflecken und Mandibel bis auf das braune Ende; Antenne schwarzbraun, unten braun, zweites Geifselglied wenig länger als 3 und $=4$. Area punktiert, fast matt. Abdomen fein und dicht punktiert, matt, Segment $1-3$ braun, fast schwarz behaart, die Segmentränder stellenweise mit schmalen gelblichen Fransenbinden, 4-7 goldgelb glänzend behaart. Ventralsegment gelbbraun bis rotbraun, ebenso behaart, 4 lappig verlängert und hier besonders dicht filzig behaart, 5 tief ausgerandet und eigenartig borstig behaart. Beine rotgelb, goldgelb behaart, Metatarsus der Länge nach gerippt, aufsen mit rotgelber Mähne (wie bei Centris), Calcar rotbraun. Flügel getrübt, Adern schwarzbraun, Tegulae rotbraun. L. $10-11 \mathrm{~mm}$, Br. $4-4^{1 / 2} \mathrm{~mm}$.

5 ๙ im Museum Hamburg, von Dr. Behn gekauft. - Neu-GuineaArchipel. 
Anthophora flammeo-zonata Dours. or 우.

Der A. zonata F. verwandt, aber rotgelb behaart, Segment $1-3$ glänzend goldgelb bandiert, 4-6 ganz goldgelb befilzt.

ㅇ. Schwarz, rotgelb behaart, Kopf und Thorax dicht runzlig punktiert, matt, Gesicht gelb gezeichnet wie bei A. zonata, Clypeus grob runzlig punktiert, Antenne braun, unten rotgelb; Area punktiert, etwas glänzend. Abdomen fein punktiert, kurz sparsam schwarz behaart, Segment 1 an der Basis sparsam gelblich behaart, Segment 1-4 mit glänzend goldgelber Randbinde, 3-4 auch auf der Scheibe mehr oder weniger gelbbraun befilzt, 5 lang rotgelb behaart, 6 mit roter Analplatte. Ventralsegmente rotbraun bis schwarzbraun, grob punktiert, gelblich gefranst. Beine rotbraun; gelbbraun behaart, Scopa rotgelb, Calcar schwarzbraun. Flügel getrübt, Adern schwarzbraun, Tegulae rotbraun. L. $11-12 \mathrm{~mm}, \mathrm{Br} .5 \mathrm{~mm}$.

$\sigma^{7}$ wie + , auch das Gesicht wenig mehr gelb gezeichnet, höchstens die schwarzen Basalflecken des Clypeus etwas kleiner; Segment 1-5 goldgelb bandiert, 5-7 gelblich befilzt; Ventralsegment 5 schwach ausgerandet, 6 vor dem Ende jederseits mit Grübchen, daher schwach gekielt. - L. $10-11 \mathrm{~mm}, \mathrm{Br} .4^{1} /_{2} \mathrm{~mm}$. or $q$ zahlreich (aus Mus. Go deffroy Nr. 4313) im Museum Hamburg von Neu-Pommern; von Dr. Behn gekauft. - NeuGuinea-Archipel.

Auch 3 우 von Sapit auf Lombok, $700 \mathrm{~m}$ hoch im AprilMai, Fruhstorfer leg. Nach Dours von Sumatra. - SundaArchipel.

Bestimmungstabelle für die mit Megachileglaberrima verwandten Arten in Australien.

(Meg. argentata F. - Gruppe.)

ㅇ.

1. Segment 6 schwarz behaart.

Segment 6 weifs behaart.

M. australiaca D. T., M. ordinaria Sm., M. macularis D. T.

2. Kopf und Thorax einfarbig weils behaart . . . . 3 . Kopf und Thorax schwarz behaart, mit gelblich behaarter

Umrandung des Mesonotum und Scutellum, sowie sonstiger
Flecken.
$M$. chyzeri Friese.

3. Kopf dick, mehr kubisch, Clypeus mitten rund ausgerandet, Körper zylindrisch, Eriades-artig. M. erimae Mocs. Kopf flach, wie gewöhnlich, Körper flachgedrückt . . 4.

4. Scopa weifs, nur auf Segment 6 schwarz, Clypeus abgestutzt, undeutlich krenuliert; Ost-Australien. M. glaberrima Friese. 
Scopa weifs, auf Segment 5-6 und an den Seiten von 2-4 schwarz, Clypeus schwach ausgerandet, nicht krenuliert; Neu-Guinea und Archipel. $M$. minutula Friese.

\section{Megachile glaberrima n. sp. 주은}

Wie Meg. argentata F. aus Europa, aber im $q$ Analsegment schwarz behaart, Scopa weils auf Segment 6 schwarz, im $\sigma^{7}$ mit verdickten braunen Tarsen I, Coxa I mit kurzem stumpfen Griffel bewehrt.

․ Schwarz, weifs behaart, Kopf und Thorax dicht und fein runzlig punktiert, Gesicht dicht weifs behaart, Clypeus grob gerunzelt, gerade abgestutzt, undeutlich krenuliert, Mandibel grofs, deutlich vierzähnig, Antenne schwarz, unten braun, zweites Geifselglied $=1$, und kürzer als 3; Area des Mittelsegments äufserst fein gerunzelt, matt. Abdomen fein punktiert, Segment $1-\mathbf{5}$ mit schmalen, weifsen Endfransen, 6 schwarz behaart, Scopa weifs, nur auf der Endhälfte vom Segment 6 schwarz, Ventralsegmente 2-5 stärker weifs gefranst und durch die Scopa durchscheinend. Beine schwarz, weifs behaart, Metatarsus von Tibienbreite, Calcar gelbbraun. Flügel hyalin, mit getrübtem Rande, Adern braun, Tegulae schwarz, punktiert. - L. $9-10 \mathrm{~mm}$, Br. $3^{1 / 4}-3^{1 / 2} \mathrm{~mm}$.

$\sigma^{7}$ wie $q$, Gesicht lang gelb behaart, Mandibel dreizähnig, Antenne lang, erreichen das Scutellum, Segment 6 weifsfilzig, Endrand schwach ausgebuchtet; Ventralsegment 5 häutig und braun. Tarsen I verdickt, schwarzbraun, nicht besonders behaart, Coxa I mit kurzem stumpfen Griffel bewehrt. - L. $8 \mathrm{~mm}$, Br. $3 \mathrm{~mm}$.

$\sigma^{\pi}+$ mehrfach von Queensland (Cairns, Mackay, Curanda), wo die Tierchen im September - November auf Heliotrop flogen. Auch in N.-S.-Wales bei Manila im Dezember. - Ost-Australien.

\section{Megachile minutula n. sp. ․․}

Der $M$. argentata F. aus Europa verwandt, sonst wie $M$. glaberrima Friese aus Australien, aber Scopa seitlich und auf Segment 5-6 schwarz.

․ Schwarz, weifslich behaart, wie M. glaberrima, aber Clypeusrana nicht gerade abgestutzt und nicht krenuliert, sondern schwach bogig ausgerandet, mit glattem Endrand, Scopa weifs, an den Seiten und am Ende breit schwarz, Tarsen innen rot beborstet. Segment 6 des Abdomens schwarz behaart. - L. $9-10 \mathrm{~mm}$, $\mathrm{Br} .3^{1 / 2} \mathrm{~mm}$.

Neu-Guinea, Stephansort, B i r o leg.; Finschhafen mehrfach, Hertle leg.; Neu-Pommern (Mus. Godeffroy Nr. 3508) mehrfach. 
Megachile fimbriventris n. sp. or우.

Der $M$. pictiventris Sm. nahestehend, aber Scopa intensiv rot, auf Segment 5-6 schwarz, o mit ebenso dichter und langer roter Bauchbehaarung.

ㅇ. Schwarz, gelbbraun behaart, Thorax oben in weiter Ausdehnung schwarz behaart; Kopf und Thorax sparsam punktiert, glänzend, Clypeus grob punktiert, mit glattem, glänzendem Mittelfeld, schwach verlängert, verjüngt, dann abgestutzt. Mandibel deutlich vierzähnig; Antenne schwarz, zweites Geifselglied $=1$ und wenig kürzer als 3 ; Nebengesicht fast weifs behaart, Scheitel schwarz behaart. Thorax oben schwarz behaart, nur die Ränder des Mesonotum gelbbraun, besonders 4 gelbliche Filzflecken vor dem Scutellum auffallend. Area fein runzlig-punktiert, ganz matt. Abdomen undeutlich sparsam punktiert, glänzend, Segment 2-5 mit sehr schmaler weifser Fransenbinde, 6 schwarz behaart, Scopa intensiv rot, auf Segment 5-6 schwarz. Beine schwarz, sparsam gelblich behaart, Tarsen schwarz behaart. Flügel getrübt, bei der Radialzelle gebräunt, Adern braun, Tegulae schwarz. L. $10-11 \mathrm{~mm}, \mathrm{Br} .4 \mathrm{~mm}$.

$\sigma^{\top}$ wie $q$, Gesicht lang weifsgelb behaart, Mandibel breit, dreizähnig, Segment 6 stumpf zweizähnig, verjüngt und dreieckig ausgeschnitten; Ventralsegmente 2-4 lang, rot und scopaartig behaart, 2 mit beulig erhabener Scheibe. Beine einfach, Tarsen III verlängert. - L. $9 \mathrm{~mm}$, Br. $3^{1} / 2 \mathrm{~mm}$.

+ mehrfach von den Fidji-Inseln (aus dem Mus. G o deffroy Nr. 2725) im Museum Hamburg von Dr. B e hn gekauft, $\sigma^{7} q$ von den Tonga-Inseln. - Ozeanien.

$$
\text { Megachile rufopilosa n. sp. or. }
$$

$\sigma^{7}$ genau wie $M$. fimbriventris gebildet, aber Segment 6 dicht rotbefilzt und Ventralsegment 2 flach, glänzend. - L. $9 \mathrm{~mm}$, Br. $3^{1} / 2 \mathrm{~mm}$.

$1 \sigma^{\top}$ von Freemantle, Dr. Frank leg. - West-Australia. Scheint die Festlandsform zu $M$. fimbriventris zu sein.

\section{Neue Bienen aus Süd-Amerika. (Hym.)}

Von Dr. H. Friese, Schwerin i. M.

Megalopta cuprea n. sp. ㅇ.

Wie $M$. idalia Sm., aber Kopf und Thorax kupferbraun, ohne grün.

ㅇ. Kupfrig braun, kurz seidenartig gelblich behaart, unregel- 


\section{$2 \mathrm{BHL}$ Biodiversity Heritage Library}

Friese, Heinrich. 1911. "Zur Bienenfauna Neuguineas und der benachbarten Gebiete. (Hym.)." Deutsche entomologische Zeitschrift 1911(4), 448-453. https://doi.org/10.1002/mmnd.48019110413.

View This Item Online: https://www.biodiversitylibrary.org/item/103379

DOI: https://doi.org/10.1002/mmnd.48019110413

Permalink: https://www.biodiversitylibrary.org/partpdf/236073

\section{Holding Institution}

Harvard University, Museum of Comparative Zoology, Ernst Mayr Library

\section{Sponsored by}

Biodiversity Heritage Library

\section{Copyright \& Reuse}

Copyright Status: Public domain. The BHL considers that this work is no longer under copyright protection.

This document was created from content at the Biodiversity Heritage Library, the world's largest open access digital library for biodiversity literature and archives. Visit BHL at https://www.biodiversitylibrary.org. 